\title{
EGFR Mutation Is Associated with Short Progression-Free Survival in Patients with Stage III Non-squamous Cell Lung Cancer Treated with Concurrent Chemoradiotherapy
}

\author{
Song Ee Park, $M D^{1}$ \\ Jae Myoung Noh, MD, PhD² \\ You Jin Kim, MD ${ }^{3}$ \\ Han Sang Lee, $\mathrm{MD}^{3}$ \\ Jang Ho Cho, MD ${ }^{3}$ \\ Sung Won Lim, MD 3 \\ Yong Chan Ahn, MD, PhD² \\ Hongryull Pyo, MD, PhD2 \\ Yoon-La Choi, MD, PhD ${ }^{4}$ \\ Joungho Han, MD, PhD ${ }^{4}$ \\ Jong-Mu Sun, MD, $P h D^{3}$ \\ Se Hoon Lee, MD, $\mathrm{PhD}^{3}$ \\ Jin Seok Ahn, MD, PhD ${ }^{3}$ \\ Keunchil Park, MD, PhD ${ }^{3}$ \\ Myung-Ju Ahn, MD, PhD
}

${ }^{1}$ Division of Hematology-Oncology, Department of Medicine, Chung-Ang University College of Medicine, Seoul, ${ }^{2}$ Department of Radiation Oncology, ${ }^{3}$ Division of Hematology-Oncology, Department of Medicine, ${ }^{4}$ Department of Pathology, Samsung Medical Center, Sungkyunkwan University School of Medicine, Seoul, Korea

\section{Purpose}

This study was conducted to evaluate the relationship between epidermal growth factor receptor (EGFR) mutation and clinical outcomes in patients with stage III non-squamous cell lung cancer treated with definitive concurrent chemoradiotherapy (CCRT).

\section{Materials and Methods}

From January 2008 to December 2013, the medical records of 197 patients with stage III non- squamous non-small cell lung cancer treated with definitive CCRT were analyzed to determine progression-free survival (PFS) and overall survival (OS) according to EGFR mutation status.

Results

Among 197 eligible patients, 81 patients were EGFR wild type, 36 patients had an EGFR mutation (exon 19 Del, n=18; L858R, n=9, uncommon [G719X, L868, T790M], n=9), and 80 patients had unknown EGFR status. The median age was 59 years (range, 28 to 80 years) and 136 patients (69.0\%) were male. The median follow-up duration was 66.5 months (range, 1.9 to 114.5 months). One hundred sixty-four patients (83.2\%) experienced disease progression. Median PFS was 8.9 months for the EGFR mutation group, 11.8 months for EGFR wild type, and 10.5 months for the unknown EGFR group ( $p=0.013$ and $p=0.042$, respectively). The most common site of metastasis in the EGFR mutant group was the brain. However, there was no significant difference in OS among the three groups (34.6 months for EGFR mutant group vs. 31.9 months for EGFR wild type vs. 22.6 months for EGFR unknown group; $\mathrm{p}=0.792$ and $\mathrm{p}=0.284)$. A total of 29 patients $(80.6 \%)$ with $E G F R$ mutation were treated with EGFR tyrosine kinase inhibitor (gefitinib, $n=24$; erlotinib, $n=3$; afatinib, $n=2$ ) upon progression.

\section{Conclusion}

EGFR mutation is associated with short PFS and the brain is the most common site of distant metastasis in patients with stage III non- squamous cell lung cancer treated with CCRT.

\section{Key words}

Non-squamous non-small cell lung cancer,

Chemoradiotherapy, Stage III, EGFR mutation, Survival

\author{
Correspondence: Myung-Ju Ahn, MD, PhD \\ Division of Hematology-Oncology, \\ Department of Medicine, Samsung Medical \\ Center, Sungkyunkwan University School \\ of Medicine, 81 Irwon-ro, Gangnam-gu, \\ Seoul 06351, Korea \\ Tel: $82-2-3410-3438$ \\ Fax: 82-2-3410-1754 \\ Email: silk.ahn@samsung.com \\ Received February 27, 2018 \\ Accepted June 10, 2018 \\ Published Online June 18, 2018 \\ *Song Ee Park and Jae Myoung Noh contributed \\ equally to this work.
}




\section{Introduction}

Concurrent chemoradiotherapy (CCRT) is the standard treatment for patients with locally advanced non-small cell lung cancer (NSCLC). CCRT is superior to radiation alone or to sequential chemoradiation in patients with unresectable stage IIIA or stage IIIB disease [1,2]. However, the majority of patients treated with CCRT develop disease recurrence and 5-year survival is only $15 \%-20 \%$ [2-4].

Epidermal growth factor receptor (EGFR) mutations are detected in approximately $40 \%$ of NSCLC from Asian patients and $10 \%-20 \%$ of NSCLC from Caucasian patients $[5,6]$. EGFR mutations are more frequently found in females, never smokers, and adenocarcinomas, regardless of ethnicity. Approximately $90 \%$ of EGFR tyrosine kinase inhibitor (TKI)-sensitizing mutations are exon 19 deletions or exon 21 L858R point mutations [7]. Several EGFR TKIs, including gefitinib, erlotinib, and afatinib have been approved for treatment of advanced EGFR-mutant NSCLC as a first-line therapy. EGFR mutation is usually associated with overexpression of EGFR. It has been reported that EGFR overexpression is negatively correlated with radiation treatment [8].

Several clinical studies recently reported that in patients with locally advanced NSCLC with EGFR mutation, locoregional recurrence rate after radiotherapy (RT) is lower than in patients with wild-type EGFR, and EGFR mutation was associated with a better response to CCRT [9]. However, the patient population in the previous studies was heterogeneous in terms of stage and surgical resection rate across EGFR mutational status. In addition, the types of EGFR mutations were not confined to deletion in exon 19 or L858R [10].

This study was conducted to evaluate the relationship between EGFR mutation and clinical outcomes in patients with stage III non-squamous cell lung cancer treated with definitive CCRT.

\section{Materials and Methods}

\section{Study design}

From January 2008 to December 2013, 334 patients with pathologically confirmed stage III NSCLC were treated with definitive CCRT at Samsung Medical Center, Korea. NSCLC stage evaluation was based on the American Joint Committee on Cancer seventh edition cancer staging manual. A total of 134 patients diagnosed with squamous cell carcinoma were excluded from further analysis. Among 200 remaining patients, three patients who did not receive CCRT or received it at half the dose of planned radiation were also excluded (Fig. 1).

The initial diagnosis of NSCLC was pathologically confirmed in all patients based on either bronchoscopy or percutaneous fine-needle biopsy. Diagnostic and staging workups included complete history and physical examination, chest computed tomography (CT), positron emission tomography-computed tomography (PET/CT) scan. Histologic diagnosis was assessed according to the World Health Organization classification. For nodal stage evaluation, endobronchial ultrasound-guided trans bronchial fine needle aspiration from a supraclavicular lymph node was performed for N2/N3 stage. EGFR (exon 18-21) mutation was detected using the peptide nucleic acid locked nucleic acid polymerase chain reaction clamp method as previously

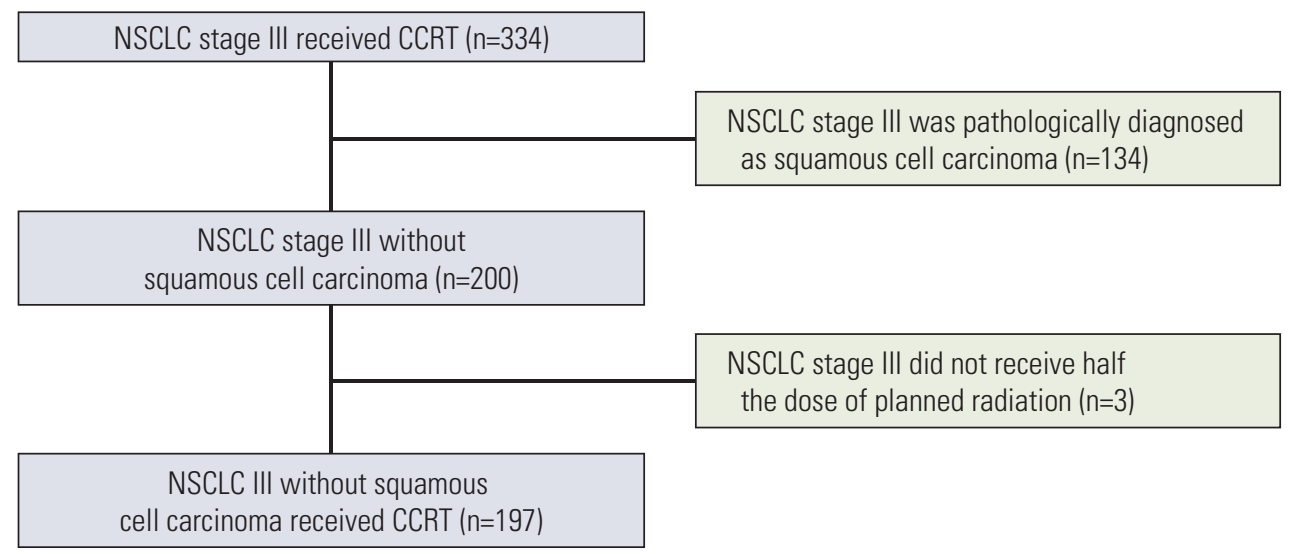

Fig. 1. Study pilot. NSCLC, non-small lung carcinoma; CCRT, concurrent chemoradiotherapy. 
described [11].

The median RT dose was 66 Gy in 33 fractions using 4-10 MV photon beams generated by a linear accelerator (Varian Medical Systems Inc., Palo Alto, CA). Simulation CT scans were typically performed in the supine position at a thickness of $2.5-5 \mathrm{~mm}$. The gross tumor volume (GTV) was defined as the volume of tumor identified based on all available clinical information, including radiologic imaging, PET scan, bronchoscopy, and mediastinoscopy.

The clinical target volume was generated by extending a 5-mm margin from the GTV, which was modified according to adjacent organs if necessary. Elective irradiation of the clinically uninvolved lymph node was not allowed. The most common concurrent chemotherapeutic regimen was docetaxel plus cisplatin. Chemotherapy consisted of six cycles of docetaxel $25 \mathrm{mg} / \mathrm{m}^{2}$ intravenously with cisplatin 25 $\mathrm{mg} / \mathrm{m}^{2}$ intravenously weekly [12]. Other CCRT regimen included paclitaxel plus platinum based chemotherapy that consisted of six cycles of paclitaxel $50 \mathrm{mg} / \mathrm{m}^{2}$ intravenously with cisplatin $25 \mathrm{mg} / \mathrm{m}^{2}$ intravenously or carboplatin area under the curve 1.5 intravenously weekly. Paclitaxel or docetaxel was given for 1 hour after chlorpheniramine $4 \mathrm{mg}$ intravenously, followed by and $\mathrm{H} 2$ blocker (ranitidine) intravenously, and dexamethasone $20 \mathrm{mg}$ intravenously. The cisplatin or carboplatin was given for 30 minutes with standard antiemetic after docetaxel. The investigator decided on docetaxel plus cisplatin or paclitaxel plus platinum (cisplatin or carboplatin) regimens. Patients received additional consolidation chemotherapy following CCRT.

Medical records were reviewed to collect patient data including age, gender, EGFR mutation status, Eastern Cooperative Oncology Group (ECOG) performance status, and smoking history. Radiological response to CCRT was evaluated by CT scan following CCRT, and was classified as a complete response (CR), partial response (PR), stable disease (SD), or progressive disease according to Response Evaluation Criteria in Solid Tumors (RECIST) ver. 1.1 [13]. The early toxicities of treatment related pneumonitis and esophagitis were graded using Common Terminology Criteria for Adverse Events ver. 4.0. The first follow-up and response evaluation were scheduled 1 month after completion of CCRT with chest CT scan. Subsequent follow-up evaluations were conducted at 3-4-month intervals thereafter, and included alternating chest $\mathrm{CT}$ and whole-body PET/CT. The primary endpoints were progression-free survival (PFS) and overall survival (OS) according to the EGFR mutation status.

\section{Statistical analysis}

The chi-square test was used to compare the response rate and recurrence rate according to EGFR mutation. PFS was measured from the start date of CCRT to the date of docu- mented treatment failure; death, disease progression, or date of censoring at last follow-up examination was considered treatment failure. OS was defined as the interval between the start date of CCRT and the date of death from any cause or the date of censoring. Rates of PFS and OS were calculated and compared using the Kaplan-Meier methods and the logrank test. In addition, multivariate analysis was conducted using Cox regression models including EGFR, stage IIIB, and disease control rate (DCR) with a p-value less than 0.05 in the univariate analysis. And, in the multivariate analysis, only the EGFR mutation group and the wild type group were analyzed, and the EGFR unknown group was excluded from the mutivariable analysis. All statistical analyses were performed using the SPSS ver. 24 (IBM Corp., Armonk, NY) software package. Variables with a p-value of $<0.05$ were considered significant.

\section{Ethical statement}

The Institutional Review Board of the Samsung Medical Center (2017-12-090) approved the study. The requirement of informed consent was waived as the study was based on the retrospective analyses of existing administrative and clinical data.

\section{Results}

\section{Patients characteristics}

Among 334 patients, 197 patients met eligibility criteria excluding squamous cell lung cancer. The main clinical characteristics of patients are summarized in Table 1. A total of 117 specimens were adequate for EGFR mutation analysis and 81 patients had unknown EGFR status. A total of 81 patients had EGFR wild type and 36 patients had EGFR mutations (exon 19 deletion, $\mathrm{n}=18$; $\mathrm{L} 858 \mathrm{R}$ in exon $21, \mathrm{n}=9$; uncommon mutation [G719X, L868, T790M], n=9). The median age was 59 years (range, 28 to 80 years) and 136 patients $(69.0 \%)$ were male. Regarding stage, 152 patients (77.2\%) had stage IIIB NSCLC and $45(22.8 \%)$ patients had stage IIIA NSCLC. A total of 131 patients $(66.5 \%)$ were current/ former smoker and 183 patients $(92.9 \%$ ) had ECOG performance status $0-1$. Regarding chemotherapy, 128 patients $(65.0 \%)$ received a docetaxel plus cisplatin regimen, 56 patients $(28.4 \%)$ received a paclitaxel plus platinum regimen, eight patients (4.1\%) received an etoposide plus cisplatin regimen, and five patients $(2.5 \%)$ received other regimens. 
Table 1. Baseline characteristics according to EGFR mutation

\begin{tabular}{|c|c|c|c|c|}
\hline Characteristic & $\begin{array}{c}\text { Total } \\
(\mathbf{n}=\mathbf{1 9 7})\end{array}$ & $\begin{array}{l}\text { EGFR mutation } \\
\qquad(\mathrm{n}=36)\end{array}$ & $\begin{array}{c}\text { EGFR wild } \\
\quad(n=81)\end{array}$ & $\begin{array}{l}\text { Unknown } \\
\qquad(\mathrm{n}=80)\end{array}$ \\
\hline Age (yr) & $59.0(28-80)$ & $52.0(39-70)$ & $60.0(28-78)$ & $63.0(31-80)$ \\
\hline$\geq 60$ & $98(49.7)$ & $9(25.0)$ & $41(50.6)$ & $48(60.0)$ \\
\hline \multicolumn{5}{|l|}{ Sex } \\
\hline Male & $136(69.0)$ & $13(36.1)$ & $59(72.8)$ & $64(80.0)$ \\
\hline Female & $61(31.0)$ & $23(63.9)$ & $22(27.0)$ & $16(20.0)$ \\
\hline \multicolumn{5}{|l|}{ Smoking status } \\
\hline Never smoker & $66(33.5)$ & $24(66.7)$ & $24(29.6)$ & $18(22.5)$ \\
\hline Current/Former smoker & $131(66.5)$ & $12(33.3)$ & $57(70.4)$ & $62(77.5)$ \\
\hline \multicolumn{5}{|l|}{ ECOG performance status } \\
\hline $0-1$ & $183(92.9)$ & $35(97.2)$ & $77(92.8)$ & $73(91.2)$ \\
\hline 2 & $14(7.1)$ & $1(2.8)$ & $6(7.2)$ & $7(8.8)$ \\
\hline \multicolumn{5}{|l|}{ Clinical T classification } \\
\hline cT1-2 & $116(58.9)$ & $22(61.1)$ & $46(56.8)$ & $48(60.0)$ \\
\hline cT3-4 & $81(41.1)$ & $14(38.9)$ & $35(43.2)$ & $32(40.0)$ \\
\hline \multicolumn{5}{|l|}{ Clinical node involvement } \\
\hline N1-2 & $62(31.5)$ & $8(22.2)$ & $24(29.6)$ & $30(37.5)$ \\
\hline N3 & $135(68.5)$ & $28(77.8)$ & $57(70.4)$ & $50(62.5)$ \\
\hline \multicolumn{5}{|l|}{ Stage } \\
\hline IIIA & $45(22.8)$ & $5(13.9)$ & $15(18.5)$ & $25(31.3)$ \\
\hline IIIB & $152(77.2)$ & $31(86.1)$ & $66(81.5)$ & $55(68.7)$ \\
\hline \multicolumn{5}{|l|}{ Pathology } \\
\hline Adenocarcinoma & $189(95.9)$ & $36(100)$ & $78(96.3)$ & $75(93.8)$ \\
\hline Large cell carcinoma & $5(2.5)$ & - & $1(1.2)$ & $4(5.0)$ \\
\hline Adenosquamous carcinoma & $3(1.5)$ & - & $2(2.5)$ & $1(1.3)$ \\
\hline \multicolumn{5}{|l|}{ EGFR mutation } \\
\hline Deletion in exon 19 & - & $18(50.0)$ & - & - \\
\hline L858R & - & $9(25.0)$ & - & - \\
\hline Uncommon & - & $9(25.0)$ & - & - \\
\hline \multicolumn{5}{|l|}{ Regimen of CCRT } \\
\hline Docetaxel+cisplatin & $128(65.0)$ & $22(61.1)$ & $47(58.0)$ & $59(73.8)$ \\
\hline Paclitaxel+platinum & $56(28.4)$ & $10(27.8)$ & $28(34.6)$ & $18(22.5)$ \\
\hline Etoposide+cisplatin & $8(4.1)$ & $1(2.8)$ & $4(4.9)$ & $3(3.7)$ \\
\hline Others & $5(2.5)$ & $3(8.3)$ & $2(2.5)$ & 0 \\
\hline Complete radiation & $190(96.4)$ & $36(100)$ & $79(97.5)$ & $75(93.8)$ \\
\hline Radiation (Gy) & $6,600(1,800-7,400)$ & $6,600(6,200-7,400)$ & $6,600(4,400-7,400)$ & $6,600(1,800-7,400)$ \\
\hline Follow-up duration (mo) & 66.5 & 66.8 & 66.5 & 64.3 \\
\hline
\end{tabular}

Values are presented as median (range) or number (\%). EGFR, epidermal growth factor receptor; ECOG, Eastern Cooperative Oncology Group; CCRT, concurrent chemoradiotherapy.

\section{Response to CCRT}

In the EGFR mutation group, one patient $(2.8 \%)$ had $\mathrm{CR}$, 25 patients $(69.4 \%)$ had $P R$, six patients $(16.7 \%)$ had SD, and four patients $(11.1 \%)$ had progression following CCRT (Table 2). With EGFR wild type, 15 patients $(18.1 \%)$ had CR, 61 patients $(75.3 \%)$ had PR, three patients $(3.7 \%)$ had SD, and two patients $(2.5 \%)$ had progression following CCRT. There was a significant difference in overall response rate to CCRT between EGFR mutant and EGFR wild type group ( $72.2 \%$ vs. $93.8 \%, \mathrm{p}<0.001)$.

\section{PFS and OS}

The median follow-up duration was 66.5 months (range, 1.9 to 114.5 months). One hundred sixty four patients (83.2\%) 
Table 2. Response according to EGFR mutation during concurrent chemoradiotherapy

\begin{tabular}{|c|c|c|c|c|c|}
\hline Efficacy of CCRT & $\begin{array}{c}\text { Total } \\
(n=197)\end{array}$ & $\begin{array}{l}\text { EGFR mutation } \\
\qquad(\mathrm{n}=36)\end{array}$ & $\begin{array}{c}\text { EGFR wild } \\
\qquad(\mathrm{n}=\mathbf{8 1})\end{array}$ & $\begin{array}{l}\text { Unknown } \\
\qquad(\mathrm{n}=80)\end{array}$ & p-value \\
\hline \multicolumn{6}{|l|}{ CCRT response } \\
\hline $\mathrm{CR}$ & $33(16.8)$ & $1(2.8)$ & 15 (18.5) & $17(21.2)$ & $<0.001$ \\
\hline PR & $126(64.0)$ & $25(69.4)$ & $61(75.3)$ & $40(50.0)$ & \\
\hline SD & $24(12.2)$ & $6(16.7)$ & $3(3.7)$ & $15(18.8)$ & \\
\hline PD & $14(7.0)$ & 4 (11.1) & $2(2.5)$ & $8(10.0)$ & \\
\hline ORR & $159(80.7)$ & $26(72.2)$ & $76(93.8)$ & $57(71.3)$ & $<0.001$ \\
\hline DCR & $183(92.9)$ & $32(88.9)$ & $79(97.5)$ & $72(90.0)$ & 0.104 \\
\hline
\end{tabular}

Values are presented as number (\%). EGFR, epidermal growth factor receptor; CCRT, chemoradiotherapy; CR, complete response; $\mathrm{PR}$, partial response; $\mathrm{SD}$, stable disease; $\mathrm{PD}$, progressive disease; $\mathrm{ORR}$, objective response rate; $\mathrm{DCR}$, disease control rate.

A

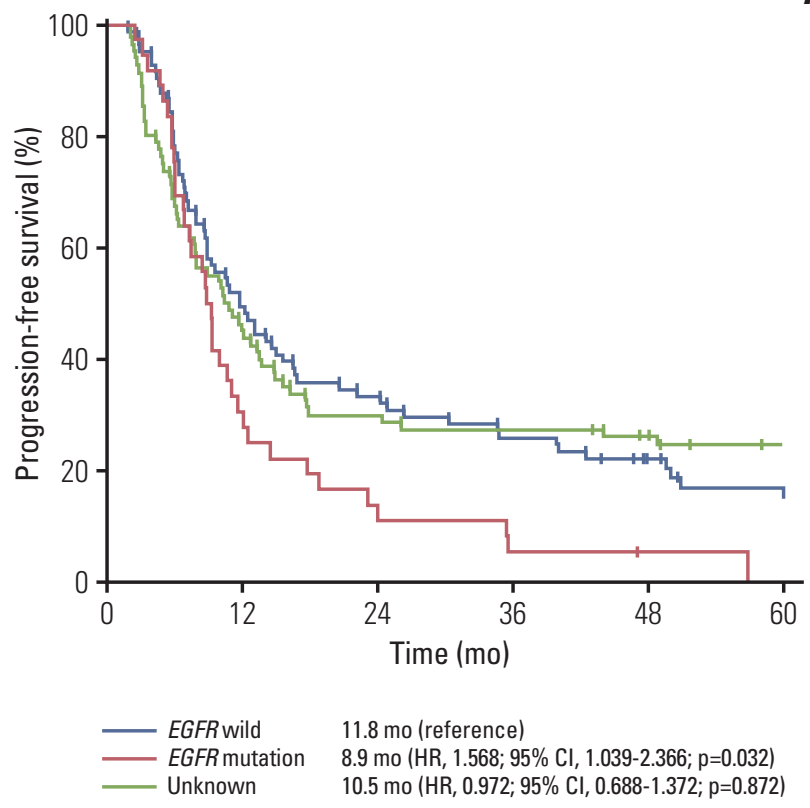

Fig. 2. Kaplan-Meier survival curves of progression-free survival (A) and overall survival (B) according to epidermal growth factor receptor (EGFR) mutation status. $\mathrm{HR}$, hazard ratio; $\mathrm{CI}$, confidence interval.

experienced disease progression. Median PFS was 8.9 months for the EGFR mutation group versus 11.8 months for EGFR wild type versus 10.5 months for EGFR unknown group (Fig. 2). The EGFR mutation group had a short PFS compared with the EGFR wild type group ( $\mathrm{p}=0.013$ ) or EGFR unknown group $(\mathrm{p}=0.042)$. By univariate analysis including EGFR mutation, DCR and stage was an independent factor for shorter PFS. By multivariate analysis, EGFR mutation group had a shorter PFS than the EGFR wild type group, although the EGFR mutation was not statistically significant

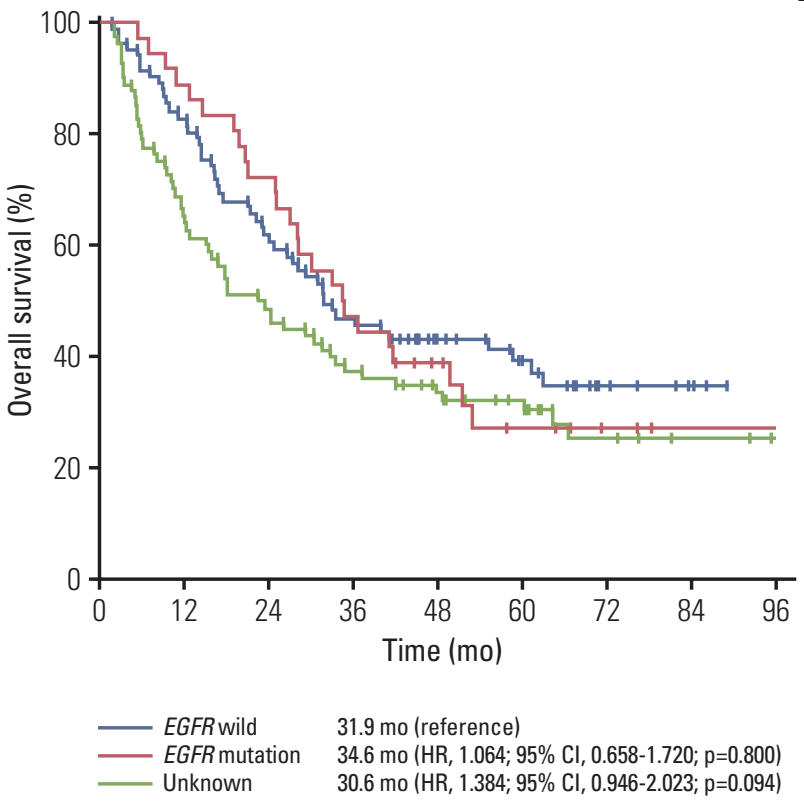


Table 3. Univariate and multivariable analyses of progression-free survival

\begin{tabular}{|c|c|c|c|c|c|c|}
\hline \multirow{2}{*}{ Variable } & \multicolumn{3}{|c|}{ Univariate analysis } & \multicolumn{3}{|c|}{ Multivariable analysis } \\
\hline & HR & $95 \% \mathrm{CI}$ & p-value & HR & $95 \% \mathrm{CI}$ & p-value \\
\hline Age $>60$ yr & 0.946 & 0.639 & 0.783 & - & - & - \\
\hline Female & 1.456 & $0.979-2.166$ & 0.063 & - & - & - \\
\hline $\mathrm{ECOG} \geq 2$ & 1.254 & $0.578-2.721$ & 0.566 & - & - & - \\
\hline Smoker & 0.691 & $0.466-1.025$ & 0.066 & - & - & - \\
\hline EGFR mutation ${ }^{\mathrm{a})}$ & 1.681 & $1.108-2.511$ & 0.015 & 1.492 & $0.968-2.229$ & 0.070 \\
\hline Stage IIIB & 1.911 & $1.098-3.326$ & 0.022 & 1.780 & $1.018-3.110$ & 0.043 \\
\hline DCR & 0.412 & $0.179-0.944$ & 0.036 & 0.542 & $0.230-1.282$ & 0.163 \\
\hline
\end{tabular}

HR, hazard ratio; CI, confidence interval; ECOG, Eastern Cooperative Oncology Group; EGFR, epidermal growth factor receptor, DCR, disease control rate. ${ }^{\text {a) } E G F R ~ m u t a t i o n ~ t y p e ~ c o m p a r e d ~ w i t h ~ E G F R ~ w i l d ~ t y p e . ~}$

A

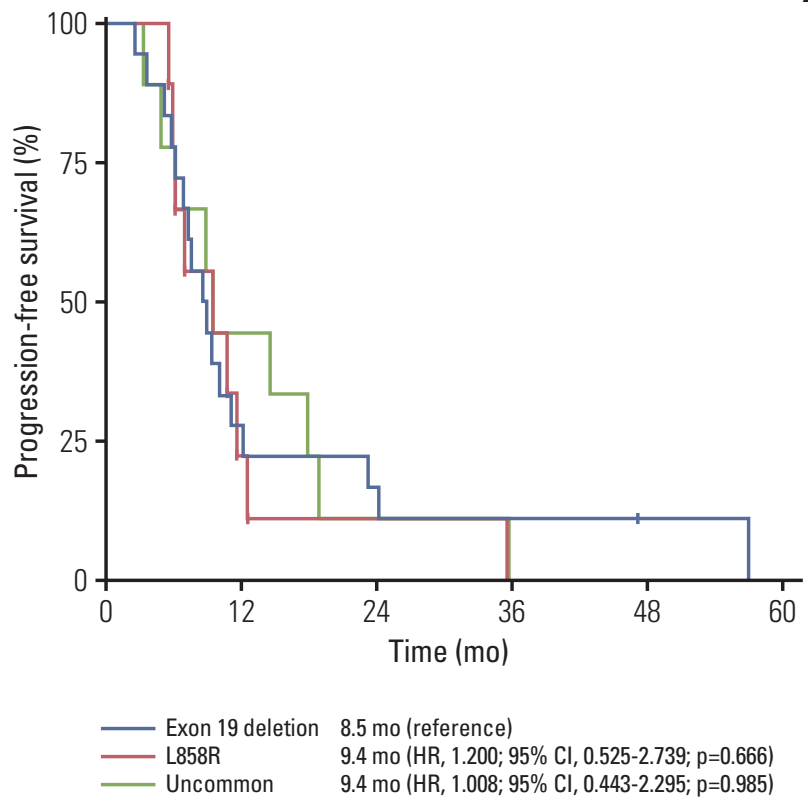

Fig. 3. Kaplan-Meier survival curves of subgroup analysis according to epidermal growth factor receptor (EGFR) mutation status. (A) Progression-free survival. (B) Overall survival. HR, hazard ratio; CI, confidence interval.

median OS according to EGFR mutation type. Twenty-nine patients $(80.6 \%)$ with EGFR mutation were treated with EGFR TKIs (gefitinib, $n=24$; erlotinib, $n=3$; afatinib, $n=2$ ) on progression. The median OS was 49.8 months for exon 19 deletions, 28.1 months for L858R and 25.1 months for uncommon EGFR mutation ( $\mathrm{p}=0.087$ ) (Fig. 3).

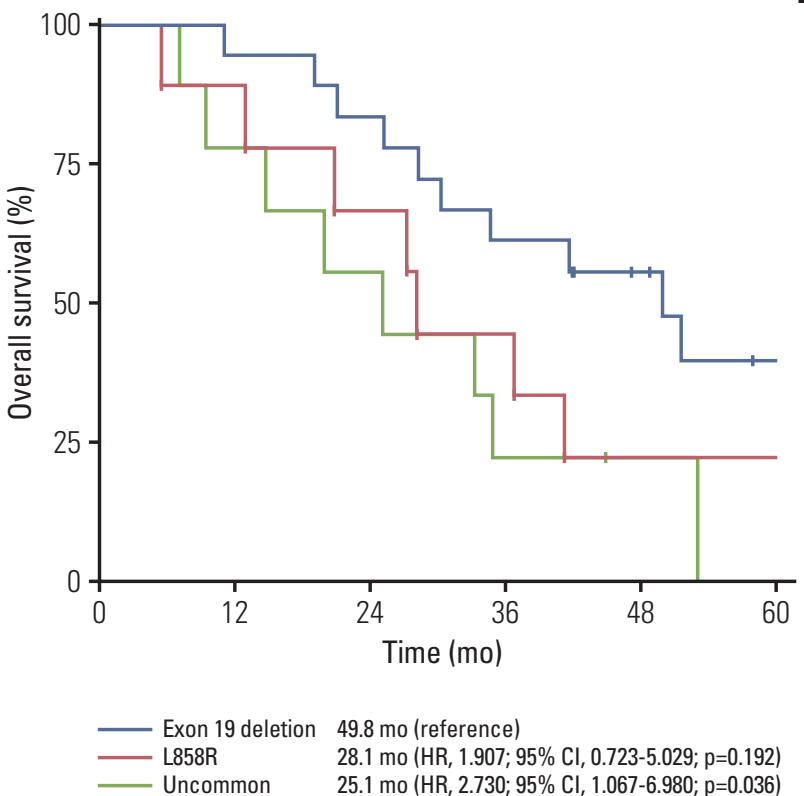

\section{Recurrence}

One hundred forty-nine patients $(75.6 \%)$ experienced tumor recurrence without death. The most common failure pattern was systemic recurrence which was observed in 99 patients $(66.4 \%)$. This was followed by loco-regional recurrence plus systemic recurrence in 32 patients $(21.5 \%)$ and loco-regional recurrence in 18 patients $(12.1 \%)$ (Table 4$)$. Among 36 patients with EGFR mutation, 33 patients (91.7\%) developed tumor recurrence without death, 23 patients 
Table 4. Recurrence rate and recurrence pattern

\begin{tabular}{|c|c|c|c|c|c|}
\hline Variable & $\begin{array}{c}\text { Total } \\
(n=198)\end{array}$ & $\begin{array}{l}E G F R \text { mutation } \\
\qquad(\mathrm{n}=36)\end{array}$ & $\begin{array}{l}\text { EGFR wild } \\
\quad(n=81)\end{array}$ & $\begin{array}{l}\text { Unknown } \\
\qquad(\mathrm{n}=80)\end{array}$ & p-value \\
\hline Recurrence rate & $149(75.6)$ & $33(91.7)$ & $64(79.0)$ & $52(65.0)$ & 0.005 \\
\hline Loco-regional recurrence & $18(12.1)$ & $3(9.1)$ & $7(10.9)$ & $8(15.4)$ & 0.793 \\
\hline Loco-regional plus systemic recurrence & $32(21.5)$ & $7(21.2)$ & $16(25.0)$ & $9(17.3)$ & \\
\hline Systemic recurrence & $99(66.4)$ & $23(69.7)$ & $41(64.1)$ & $35(67.3)$ & \\
\hline
\end{tabular}

Values are presented as number (\%). EGFR, epidermal growth factor receptor.

A

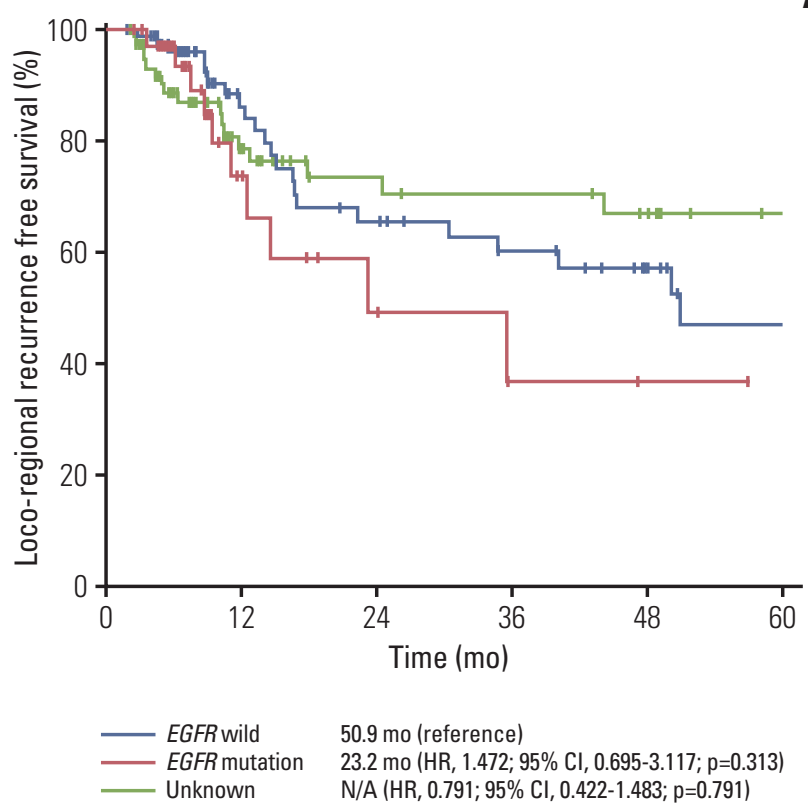

Fig. 4. Kaplan-Meier survival curves of loco-regional recurrence free survival (A) and distant metastasis free survival (B). EGFR, epidermal growth factor receptor; $\mathrm{HR}$, hazard ratio; $\mathrm{CI}$, confidence interval.

$(69.7 \%)$ had systemic recurrence, seven patients $(21.2 \%)$ had loco-regional recurrence plus systemic recurrence, and three patients $(9.1 \%)$ had loco-regional recurrence. The most common site of distant metastasis in the EGFR mutant group was the brain in nine patients followed by pleural metastasis in six patients, bone metastasis in five patients, lung to lung metastasis in five patients, distant lymph node metastasis in three patients, adrenal gland metastasis in two patients and liver metastasis in one patient. In the EGFR wild type group, pleural metastasis (15 patients, 18.5\%) was the most common metastatic lesion, followed by brain (13 patients, 16.0\%). In the EGFR unknown group, the brain was the most common metastatic site (16 patients, 20.0\%). The median distant metastasis free survival (DMFS) was 9.3 months for the EGFR mutant group versus 13.2 months for EGFR wild type versus

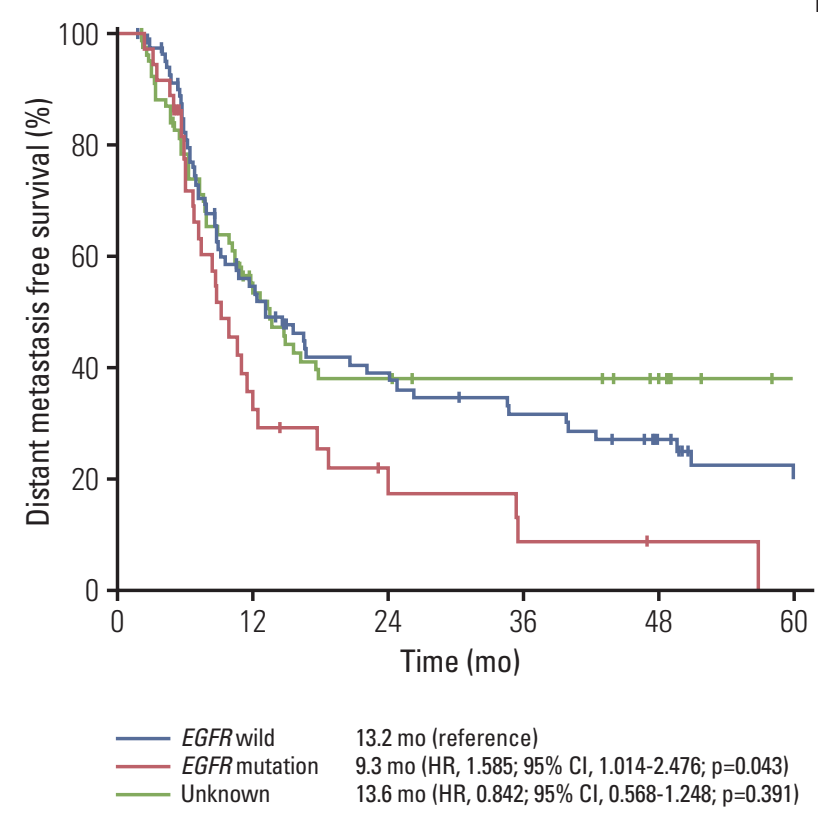

13.6 months for the EGFR unknown group. The EGFR mutation group had a short DMFS compared with the EGFR wild type group ( $\mathrm{p}=0.022)$ and EGFR unknown group $(\mathrm{p}=0.013)$. The median loco-regional recurrence free survival was 23.2 months for the EGFR mutant group versus 50.9 months for the EGFR wild type group ( $\mathrm{p}=0.184$ ) (Fig. 4).

\section{Discussion}

In this study, PFS was significantly shorter in patients with EGFR mutations compared to the EGFR wild type or unknown EGFR groups. The most common recurrence pat- 
tern was systemic with or without loco-regional recurrence, which was observed in more than $87.9 \%$ of patients. It was previously reported that EGFR mutation was associated with more frequent distant relapse and worse 5-year PFS rate after neoadjuvant CCRT followed by surgery in locally advanced mediastinoscopic N2-positive NSCLC [14]. These findings suggest that systemic control is more important in patients with locally advanced adenocarcinoma with EGFR mutations. Given the high incidence of systemic recurrence and limited clinical outcomes in EGFR mutant patients, a randomized phase III trial of maintenance gefitinib after CCRT in locally advanced NSCLC (SWOG S0023) was conducted to evaluate whether maintenance gefitinib improved clinical outcomes [15]. Unexpectedly, patients who received gefitinib as maintenance had worse survival compared to gefitinib and experienced more adverse events, although only few patients have EGFR mutation [16]. In our study, the PFS was significantly shorter in patients with EGFR mutation compared with EGFR wild type. The OS in our study did not show any significant difference between the two groups due to salvage therapy with EGFR TKI after progression. Another concern is the development of resistance to maintenance EGFR TKI. Therefore, it remains unclear whether maintenance EGFR TKI improves OS in stage III NSCLC patients after completion of CCRT.

Intriguingly, we also found that the brain was the most frequent site of distant metastasis in patients with EGFR mutation, consistent with other studies $[17,18]$. This finding suggests that prophylactic central nervous system irradiation (PCI) might benefit patients with EGFR mutation. Recently, PCI in locally advanced NSCLC after CCRT did not show any survival benefit although the relapse rate in brain decreased [19]. Thus, the role of PCI in stage III EGFR mutant NSCLC must be investigated further. In contrast, the thirdgeneration EGFR TKI osimertinib is a candidate for consolidation therapy because of its high permeability to the brain and promising central nervous system efficacy $[20,21]$. In contrast, the locoregional recurrence rate of the EGFR mutation group $(9.1 \%)$ was not significantly different from that of the EGFR wild type group (10.9\%), which is not consistent with previous studies [17] and needs further evaluation. Of note, the response rate to CCRT in the EGFR mutation group was also significantly lower than those of the EGFR wild type group. However, there was no significant difference in OS between the EGFR mutation group and the EGFR wild type group. Although no significant difference in PFS was observed according to EGFR mutation type, the median OS was longest in patients with exon 19 deletions.

Recently, a randomized phase III study of maintenance durvalumab, anti-PD-L1 immune check point inhibitor after completion of CCRT in stage III NSCLC patients (PACIFIC), demonstrated significant improvement of PFS compared to placebo (16.8 months vs. 5.6 months) [22]. In this study, only $6.1 \%$ of patients had EGFR mutations. Given that immune checkpoint inhibitors do not improve OS in EGFR mutant NSCLC by meta-analysis, there is a high possibility that durvalumab as consolidation therapy may not be beneficial to patients with EGFR mutant stage III NSCLC after completion of CCRT. Subgroup analysis in patients with EGFR mutation showed a hazard ratio of 0.76 ( $95 \%$ confidence interval, 0.35 to 1.64), suggesting patients with EGFR mutation might not benefit from maintenance durvalumab in this setting [23-25]. Although retrospective nature of analysis and single center study, it is one of the largest series of cohort patient dataset treated with CCRT along with long-term follow-up comparing EGFR mutation and wild type.

In conclusion, EGFR mutation was associated with short PFS and brain was the most common site of distant metastasis in patients with stage III non-squamous cell lung cancer treated with CCRT. To improve clinical outcome in this specific subset of patients, further study with novel agent should be investigated.

\section{Conflicts of Interest}

Conflict of interest relevant to this article was not reported.

\section{Acknowledgments}

The National Research Foundation of Korea (NRF) funded by the Ministry of Science, ICT \& Future Planning (NRF-2017M3A9G5060252) supported this research.

\section{References}

1. Dillman RO, Seagren SL, Propert KJ, Guerra J, Eaton WL, Perry MC, et al. A randomized trial of induction chemotherapy plus high-dose radiation versus radiation alone in stage III non-small-cell lung cancer. N Engl J Med. 1990;323:940-5.

2. Furuse K, Fukuoka M, Kawahara M, Nishikawa H, Takada Y,
Kudoh S, et al. Phase III study of concurrent versus sequential thoracic radiotherapy in combination with mitomycin, vindesine, and cisplatin in unresectable stage III non-small-cell lung cancer. J Clin Oncol. 1999;17:2692-9.

3. Segawa Y, Kiura K, Takigawa N, Kamei H, Harita S, Hiraki S, 
et al. Phase III trial comparing docetaxel and cisplatin combination chemotherapy with mitomycin, vindesine, and cisplatin combination chemotherapy with concurrent thoracic radiotherapy in locally advanced non-small-cell lung cancer: OLCSG 0007. J Clin Oncol. 2010;28:3299-306.

4. Yamamoto N, Nakagawa K, Nishimura Y, Tsujino K, Satouchi M, Kudo S, et al. Phase III study comparing second- and thirdgeneration regimens with concurrent thoracic radiotherapy in patients with unresectable stage III non-small-cell lung cancer: West Japan Thoracic Oncology Group WJTOG0105. J Clin Oncol. 2010;28:3739-45.

5. Kris MG, Johnson BE, Berry LD, Kwiatkowski DJ, Iafrate AJ, Wistuba II, et al. Using multiplexed assays of oncogenic drivers in lung cancers to select targeted drugs. JAMA. 2014;311: 1998-2006.

6. Yatabe Y, Kerr KM, Utomo A, Rajadurai P, Tran VK, Du X, et al. EGFR mutation testing practices within the Asia Pacific region: results of a multicenter diagnostic survey. J Thorac Oncol. 2015;10:438-45.

7. Han JY, Kim SH, Lee YS, Lee SY, Hwang JA, Kim JY, et al. Comparison of targeted next-generation sequencing with conventional sequencing for predicting the responsiveness to epidermal growth factor receptor-tyrosine kinase inhibitor (EGFR-TKI) therapy in never-smokers with lung adenocarcinoma. Lung Cancer. 2014;85:161-7.

8. Nguyen KS, Neal JW. First-line treatment of EGFR-mutant non-small-cell lung cancer: the role of erlotinib and other tyrosine kinase inhibitors. Biologics. 2012;6:337-45.

9. Li F, Bai H, Li X, Wu M, Yu R, Shi A, et al. Role of EGFR mutation status in patients with stage III non-squamous nonsmall cell lung cancer treated with chemoradiotherapy. Zhongguo Fei Ai Za Zhi. 2011;14:715-8.

10. Mak RH, Doran E, Muzikansky A, Kang J, Neal JW, Baldini $\mathrm{EH}$, et al. Outcomes after combined modality therapy for EGFR-mutant and wild-type locally advanced NSCLC. Oncologist. 2011;16:886-95.

11. Tanaka T, Nagai Y, Miyazawa H, Koyama N, Matsuoka S, Sutani A, et al. Reliability of the peptide nucleic acid-locked nucleic acid polymerase chain reaction clamp-based test for epidermal growth factor receptor mutations integrated into the clinical practice for non-small cell lung cancers. Cancer Sci. 2007;98:246-52.

12. Bradley JD, Paulus R, Komaki R, Masters G, Blumenschein G, Schild S, et al. Standard-dose versus high-dose conformal radiotherapy with concurrent and consolidation carboplatin plus paclitaxel with or without cetuximab for patients with stage IIIA or IIIB non-small-cell lung cancer (RTOG 0617): a randomised, two-by-two factorial phase 3 study. Lancet Oncol. 2015;16:187-99.

13. Eisenhauer EA, Therasse P, Bogaerts J, Schwartz LH, Sargent D, Ford R, et al. New response evaluation criteria in solid tumours: revised RECIST guideline (version 1.1). Eur J Cancer.
2009;45:228-47.

14. Ahn HK, Choi YL, Han JH, Ahn YC, Kim K, Kim J, et al. Epidermal growth factor receptor mutation and treatment outcome of mediastinoscopic N2 positive non-small cell lung cancer patients treated with neoadjuvant chemoradiotherapy followed by surgery. Lung Cancer. 2013;79:300-6.

15. Kelly K, Chansky K, Gaspar LE, Albain KS, Jett J, Ung YC, et al. Phase III trial of maintenance gefitinib or placebo after concurrent chemoradiotherapy and docetaxel consolidation in inoperable stage III non-small-cell lung cancer: SWOG S0023. J Clin Oncol. 2008;26:2450-6.

16. Bhutani M, Pathak AK, Mao L. SWOG S0023: what meets the eye may be only half the truth. J Clin Oncol. 2008;26:4848-9.

17. Tanaka K, Hida T, Oya Y, Oguri T, Yoshida T, Shimizu J, et al. EGFR mutation impact on definitive concurrent chemoradiation therapy for inoperable stage III adenocarcinoma. J Thorac Oncol. 2015;10:1720-5.

18. Akamatsu H, Kaira K, Murakami H, Serizawa M, Koh Y, Ono A, et al. The impact of clinical outcomes according to EGFR mutation status in patients with locally advanced lung adenocarcinoma who recieved concurrent chemoradiotherapy. Am J Clin Oncol. 2014;37:144-7.

19. Gore EM, Bae K, Wong SJ, Sun A, Bonner JA, Schild SE, et al. Phase III comparison of prophylactic cranial irradiation versus observation in patients with locally advanced non-small-cell lung cancer: primary analysis of radiation therapy oncology group study RTOG 0214. J Clin Oncol. 2011;29:272-8.

20. Mok TS, Wu YL, Ahn MJ, Garassino MC, Kim HR, Ramalingam SS, et al. Osimertinib or platinum-pemetrexed in EGFR T790M-positive lung cancer. N Engl J Med. 2017;376:629-40.

21. Ballard P, Yates JW, Yang Z, Kim DW, Yang JC, Cantarini M, et al. Preclinical comparison of osimertinib with other EGFRTKIs in EGFR-mutant NSCLC brain metastases models, and early evidence of clinical brain metastases activity. Clin Cancer Res. 2016;22:5130-40.

22. Antonia SJ, Villegas A, Daniel D, Vicente D, Murakami S, Hui $\mathrm{R}$, et al. Durvalumab after chemoradiotherapy in stage III nonsmall-cell lung cancer. N Engl J Med. 2017;377:1919-29.

23. Borghaei H, Paz-Ares L, Horn L, Spigel DR, Steins M, Ready $\mathrm{NE}$, et al. Nivolumab versus docetaxel in advanced nonsquamous non-small-cell lung cancer. N Engl J Med. 2015;373:162739.

24. Herbst RS, Baas P, Kim DW, Felip E, Perez-Gracia JL, Han JY, et al. Pembrolizumab versus docetaxel for previously treated, PD-L1-positive, advanced non-small-cell lung cancer (KEYNOTE-010): a randomised controlled trial. Lancet. 2016;387: 1540-50.

25. Rittmeyer A, Barlesi F, Waterkamp D, Park K, Ciardiello F, von Pawel J, et al. Atezolizumab versus docetaxel in patients with previously treated non-small-cell lung cancer (OAK): a phase 3, open-label, multicentre randomised controlled trial. Lancet. 2017;389:255-65. 